\title{
The Subjective Musculoskeletal Symptoms of Dental Hygienists According to Their Treatment Posture
}

\author{
hyo jeong kim \\ Department of Dental Hygiene, Andong Science College, Andong, Korea
}

\begin{abstract}
Objective: A self-administered questionnaire was conducted with three hundred dental hygienists. A total of three hundred questionnaire copy is collected. The copies with insincere answers or errors were excluded, and finally two hundred and 61 copies were used.
\end{abstract}

Methods: The collected data were analyzed with SPSS 12.0 (SPSS Inc., Chicago, IL, USA) program. The frequency and percentage of the study subjects' subjective pains of musculoskeletal symptoms were calculated according to the characteristics of their work conditions and their body regions related to work. In order to analyze relations between the subjective musculoskeletal symptom in the each one of body regions, and work conditions and treatment posture, Pearson's Correlation Analysis was conducted.

Results: 1) Regarding their daily treatment hours, those working 2 to 4 hours accounted for the highest rate (46.4\%)-Their subjective musculoskeletal symptoms were found in the order of the shoulders (76.0\%), the neck (75.2\%), and the waist (71.1\%). 2) Regarding the use of their hands for treatment, those using both hands accounted for $44.4 \%$-Their subjective musculoskeletal symptoms were found in the order of the shoulders $(76.7 \%)$, the neck $(70.7 \%)$, the waist $(64.7 \%)$, and the wrists $(60.3 \%)$. Subjective musculoskeletal symptoms on the wrists were more found. 3) Regarding dental hygienists' operation position for treatment, those in the 9 o'clock position accounted for $63.6 \%$-Their subjective musculoskeletal symptoms were found in the order of the shoulders $(80.1 \%)$, the neck $(75.9 \%)$, and the legs/feet $(66.3 \%)$.

Conclusion: Most dental hygienist had the problem with waist and shoulder posture for direct treatment, and suffered subjective musculoskeletal symptoms mostly on the shoulders, the neck, and the waist in order.

Keywords: dental hygienists, musculoskeletal disorder, symptoms, pain

Corresponding author hyo jeong kim

E-mail: med8097@hotmail.com

(iD) https://orcid.org/0000-0002-5407-2718

Received December 1, 2021, Revised December 21, 2021, Accepted December 24, 2021

\section{Introduction}

With the enlargement and specialization of dentistry, the work conditions of dental hygienists have more been diversified, and thereby their work types have been expanded. Dental hygienists working in dental institutions or clinics are easily exposed to musculoskeletal disorders, considering their work conditions and treatment posture, and consequently more attention has been paid to them [1-3].

Copyright (C) 2021. Korean Academy of Preventive Dentistry.

This is an Open Access article distributed under the terms of the Creative Commons Attribution Non-Commercial License (http://creativecommons.org/licenses/ by-nc/4.0) which permits unrestricted non-commercial use, distribution, and reproduction in any medium, provided the original work is properly cited. 
The purpose of this study is to find the work conditions and musculoskeletal disorders of dental hygienist working at dental hospitals or clinics, and to provide a fundamental research material for designing measures and preventive programs to resolve the problems [4-5].

\section{Materials and Methods}

\section{Subjects}

A self-administered questionnaire (from November 1, 2017 to May 30, 2018, from February 1, 2021 to Mar 31, 2021) was conducted with three hundred dental hygienists. A total of three hundred questionnaire copy is collected. The copies with insincere answers or errors were excluded, and finally two hundred and 61 copies were used.

\section{Methods}

The collected data were analyzed with SPSS 12.0 (SPSS Inc., Chicago, IL, USA) program. The frequency and percentage of the study subjects' subjective pains of musculoskeletal symptoms were calculated according to the characteristics of their work conditions and their body regions related to work.

In order to analyze relations between the subjective musculoskeletal symptom in the each one of body regions, and work conditions and treatment posture. Pearson's Correlation Analysis was conducted.

\section{Results}

\section{General characteristics}

Regarding a type of dental institution, $83.1 \%$ worked at dental clinics, $13.8 \%$ at dental hospitals, and $3.1 \%$ at dental university hospitals. Therefore, it was found that most of them worked at dental clinics. Regarding the body regions related to the subjective musculoskeletal pains of those working at dental clinics, $77.9 \%$ had subjective pains on the shoulders, $75.1 \%$ on the neck, and $68.7 \%$ on the waist.

Regarding a type of position, $68.2 \%$ were in the position of dental hygienist. Regarding the body regions related to their subjective musculoskeletal pains, $77.5 \%$ had subjective pains on the shoulders, $73.0 \%$ on the neck, and $63.5 \%$ on the legs [6].

Regarding service career, $72.4 \%$ worked for less than 5 years. Regarding the body regions related to the subjective musculoskeletal pains of those with a career of less than 2 years, $77.5 \%$ had subjective pains on the shoulders, $75.5 \%$ on the neck, and $74.5 \%$ on the waist. As for those with a career of 3 to 5 years, $78.9 \%$ subjective pains on the shoulders, $70.5 \%$ on the waist, and $68.4 \%$ on the neck. As for those with a career of more than 5 years, $77.8 \%$ had subjective pains on the shoulders, $76.4 \%$ on the neck, and $61.1 \%$ on the waist. Therefore, the subjective pains on the shoulders according to their service career accounted for the highest percentage [7].

Regarding a type of work, $90.0 \%$ worked in treatment room, $44.4 \%$ in patient consultation room, and $30.2 \%$ in help desk. Regarding the body regions related to the subjective musculoskeletal pains of those working in treatment room, 79.1\% had subjective pains on the shoulders, $73.6 \%$ on the neck, and $71.5 \%$ on the waist. As for those working in patient consultation room, $80.2 \%$ subjective pains on the shoulders, $72.4 \%$ on the neck, and $66.4 \%$ on the waist [8]. Table 1 shows the distribution made according to the study subject's general characteristics.

\section{Characteristics of treatment}

Regarding of dental hygienists' main medical subjects, $87.3 \%$ were involved in scaling, $78.5 \%$ in dental prosthetic treatment, $59.7 \%$ in oral surgery (implant and periodontal treatment, 50.9\% in orthodontic treatment, $31.4 \%$ in oral health education, and $22.2 \%$ in prevention (sealant, etc.).

With regard to weekly scaling treatment, $49.0 \%$ performed the treatment 1 to 5 times, $32.1 \% 6$ to 10 times, and $18.7 \%$ more than 11 times. Regarding the body regions related to the subjective musculoskeletal pains of those serving scaling 1 to 5 times, $78.9 \%$ had subjective pains on the shoulders, $71.1 \%$ on the neck, $73.8 \%$ on the waist, and $60.9 \%$ on the wrists. As for those serving scaling 6 to 10 times, $82.1 \%$ had subjective pains on the shoulders, $76.2 \%$ on the neck, and $73.8 \%$ on the waist. As for those serving scaling more than 11 times, $73.5 \%$ had subjective pains on the neck, $69.4 \%$ on the shoulders, and $69.4 \%$ on the waist [9].

Regarding the number of dental hygienists working in dental institutions, $38.3 \%$ had 4 to 6 dental hygienists, $24.9 \%$ had 1 to 3 dental hygienists, $15.7 \%$ had 7 to 9 dental hygienists, and $21 \%$ had more than 10 dental hygienists. Table 2 shows the distribution made according to the study subject's characteristics of treatment.

\section{Characteristics of treatment posture}

With regard to daily direct treatment hours, $14.9 \%$ had the treatment less than 2 hours, $46.4 \% 2$ to 4 hours, $38.7 \%$ and more than 4 hours [10].

Regarding the body regions related to the subjective musculoskeletal symptoms of those who had the treatment less than 2 hours, $87.2 \%$ had subjective musculoskeletal symptoms on the shoulders, $66.7 \%$ on the neck, and $76.9 \%$ on the waist. As for those who had the treatment 2 to 4 hours, $76.0 \%$ had subjective musculoskeletal symptoms on the shoulders, $75.2 \%$ on 
Table 1. General characteristics

\begin{tabular}{|c|c|c|c|c|c|c|c|c|c|c|c|}
\hline & Variable & $\mathrm{N}$ & Eye & Neck & Shoulder & Arm/elbow & Hand & Wrist & Finger & Waist & Leg/foot \\
\hline \multirow{4}{*}{$\begin{array}{l}\text { Type of } \\
\text { dental } \\
\text { institution }\end{array}$} & Dental clinics & 217 & $89(41.0)$ & $163(75.1)$ & 169 (77.9) & $70(32.3)$ & $80(36.9)$ & $140(64.5)$ & 75 (34.6) & $149(68.7)$ & $130(59.9)$ \\
\hline & Dental hospitals & 36 & $17(47.2)$ & 23 (63.9) & 29 (80.6) & 7 (19.4) & $12(33.3)$ & 19 (52.8) & 7 (19.4) & $26(72.2)$ & $23(63.9)$ \\
\hline & $\begin{array}{l}\text { Dental university } \\
\text { hospitals }\end{array}$ & 8 & & & & & & & & & \\
\hline & $p$-value & & 0.759 & 0.292 & & 0.277 & & & 0.1 & & 0.897 \\
\hline \multirow{4}{*}{$\begin{array}{l}\text { Service } \\
\text { career }(\mathrm{yr})\end{array}$} & $\leq 2$ & 94 & $38(40.4)$ & $71(7$ & $73(77.7)$ & 30 & 40( & $61(64.9)$ & 36( & 70( & 63( \\
\hline & $3-5$ & 95 & 39 (41.1) & 65 (68.4) & 75 (78.9) & $25(26.3)$ & $32(33.7)$ & $60(63.2)$ & 29 (30.5) & 67 (70.5) & $52(54.7)$ \\
\hline & $\geq 5$ & 72 & $32(44.4)$ & $55(76.4)$ & $56(77.8)$ & $25(3$ & $24(33.3)$ & $42(58.3)$ & 21 & $44(6$ & $43(59.7)$ \\
\hline & & & 0.860 & 0.419 & 0.973 & 0.4 & 0.349 & 0.677 & 0.380 & 0.172 & 0.222 \\
\hline \multirow[t]{4}{*}{ Type of work } & Treatment room & 235 & $101(43.0)$ & 173 (73.6) & $186(79.1)$ & $73(31.1)$ & 89 (37.9) & $150(63.8)$ & $80(34.0)$ & $168(71.5)$ & $146(62.1)$ \\
\hline & Desk & 79 & & & & & & 47 (59.5) & & & 45 (57.0) \\
\hline & $\begin{array}{l}\text { Patient consultation } \\
\text { room }\end{array}$ & 116 & $42(36.2)$ & $84(72.4)$ & $93(80.2)$ & $35(30.2)$ & 37 (31.9) & $64(55.2)$ & 34 (29.3) & 77 (66.4) & $64(55.2)$ \\
\hline & Clinic management & 25 & $7(28.0)$ & $14(56.0)$ & $15(60.0)$ & $3(12.0)$ & $5(20.0)$ & $12(48.0)$ & $3(12.0)$ & $10(40.0)$ & $9(36.0)$ \\
\hline
\end{tabular}

Values are presented as number $(\%)$.

Table 2. Characteristics of treatment

\begin{tabular}{clcccccccccc}
\hline \multicolumn{1}{c}{ Variable } & $\mathrm{N}$ & Eye & Neck & Shoulder & Arm/elbow & Hand & Wrist & Finger & Waist & Leg/foot \\
\hline Main & Prosthetic & 205 & $92(84.4)$ & $153(80.5)$ & $158(77.8)$ & $67(84.8)$ & $80(84.2)$ & $131(80.9)$ & $69(81.2)$ & $144(80.0)$ & $128(81.5)$ \\
medical & Orthodontic & 133 & $51(46.8)$ & $99(52.1)$ & $104(51.2)$ & $43(54.4)$ & $46(48.4)$ & $82(50.6)$ & $39(45.9)$ & $95(52.8)$ & $82(52.2)$ \\
subjects & Periodontal & 172 & $78(71.6)$ & $129(67.9)$ & $128(63.1)$ & $57(72.2)$ & $69(72.6)$ & $112(69.1)$ & $60(70.6)$ & $123(68.3)$ & $109(69.4)$ \\
& Oral surgery & 156 & $64(58.7)$ & $118(62.1)$ & $115(56.7)$ & $51(64.6)$ & $63(66.3)$ & $102(63.0)$ & $54(63.5)$ & $110(61.1)$ & $97(61.8)$ \\
& Scaling & 228 & $96(88.1)$ & $168(88.4)$ & $179(88.2)$ & $70(88.6)$ & $84(88.4)$ & $150(92.6)$ & $76(89.4)$ & $162(90.0)$ & $137(87.3)$ \\
& Sealant & 58 & $29(26.6)$ & $44(23.2)$ & $46(22.7)$ & $21(26.6)$ & $28(29.5)$ & $41(25.3)$ & $23(27.1)$ & $43(23.9)$ & $40(25.5)$ \\
& Preservation of & 82 & $33(30.3)$ & $56(29.5)$ & $68(33.5)$ & $26(32.9)$ & $33(34.7)$ & $56(34.6)$ & $38(44.7)$ & $55(30.6)$ & $52(33.1)$ \\
& oral health & & & & & & & & & & \\
Scaling & etc. & 5 & $2(1.8)$ & $3(1.6)$ & $5(2.5)$ & $0(0.0)$ & $0(0.0)$ & $0(0.0)$ & $1(1.2)$ & $5(2.8)$ & $3(1.9)$ \\
(1 week) & 6-10 & 128 & $58(45.3)$ & $91(71.1)$ & $101(78.9)$ & $43(33.6)$ & $49(38.3)$ & $78(60.9)$ & $41(32.0)$ & $85(66.4)$ & $82(64.1)$ \\
& $\geq 11$ & 84 & $33(39.3)$ & $64(76.2)$ & $69(82.1)$ & $23(27.4)$ & $30(35.7)$ & $58(69.0)$ & $26(31.0)$ & $62(73.8)$ & $51(60.7)$ \\
& p-value & 49 & $18(36.7)$ & $36(73.5)$ & $34(69.4)$ & $14(28.6)$ & $17(34.7)$ & $27(55.1)$ & $19(38.8)$ & $34(69.4)$ & $25(51.0)$ \\
Number of & $1-3$ & & 0.500 & 0.714 & 0.220 & 0.593 & 0.880 & 0.245 & 0.621 & 0.520 & 0.283 \\
dental & $4-6$ & 65 & $23(35.4)$ & $50(76.9)$ & $52(80.0)$ & $21(32.3)$ & $23(35.4)$ & $42(64.6)$ & $20(30.8)$ & $48(73.8)$ & $41(63.1)$ \\
hygienist & $7-9$ & 100 & $52(52.0)$ & $80(80.0)$ & $79(79.0)$ & $30(30.0)$ & $37(37.0)$ & $68(68.0)$ & $37(37.0)$ & $74(74.0)$ & $62(62.0)$ \\
& $\geq 10$ & 41 & $13(31.7)$ & $24(58.5)$ & $33(80.5)$ & $13(31.7)$ & $16(39.0)$ & $20(48.8)$ & $12(29.3)$ & $22(53.7)$ & $25(61.0)$ \\
& p-value & 55 & $21(38.2)$ & $37(67.3)$ & $40(72.7)$ & $16(29.1)$ & $20(36.4)$ & $33(60.0)$ & $17(30.9)$ & $37(67.3)$ & $30(54.5)$ \\
& & 0.060 & 0.040 & 0.741 & 0.979 & 0.985 & 0.183 & 0.744 & 0.090 & 0.778 \\
\hline
\end{tabular}

Values are presented as number $(\%)$.

the neck, and $71.1 \%$ on the waist. As for those who had the treatment more than 4 hours, $7.2 \%$ had subjective musculoskeletal symptoms on the shoulders, $73.3 \%$ on the neck, $67.3 \%$ on the wrists, and $64.4 \%$ on the waist.

Regarding the hand (s) used for treatment, $52.1 \%$ used the right hand, 3.5\% used the left hand, and 44.4\% used both hands. Regarding the body regions related to the subjective musculoskeletal symptoms of dental hygienists using their both hands for treatment, $76.7 \%$ had subjective musculoskeletal symptoms on the shoulders, $70.7 \%$ on the neck, $64.7 \%$ on the waist, and $60.3 \%$ on the waist [10]. As for dental hygienists using the right hand for treatment, $79.4 \%$ had subjective musculoskeletal symptoms on the shoulders, $75.2 \%$ on the neck, $72.8 \%$ on the waist, and $64.7 \%$ on the legs/feet. The dental hygienists using one hand had more subjective musculoskeletal symptoms than those using both hands [10-11].

Regarding dental hygienists' operation position for treatment, those in the 6 o'clock position accounted for $5.7 \%$, those in the 9 o'clock position for $63.6 \%$, those in the 12 o'clock position for $26 \%$, and others for $4.5 \%$. Regarding the body regions re- 
lated to the subjective musculoskeletal symptoms of those who had the 6 o'clock position, $73.3 \%$ had subjective musculoskeletal symptoms on the waist, and $66.7 \%$ on the neck, the shoulders, and the wrists. As for those who had the 9 o'clock position, $80.1 \%$ had subjective musculoskeletal symptoms on the shoulders, $75.9 \%$ on the neck, and $66.3 \%$ on the legs/feet. As for those who had the 12 o'clock position, $75.0 \%$ had subjective musculoskeletal symptoms on the shoulders, $67.6 \%$ on the neck, and $66.2 \%$ on the waist.

Regarding the question "do you bend down more than 15 degrees or twist your body in order for treatment?", 60.9\% answered "Yes", 31.8\% answered "Yes but not always", and $7.3 \%$ answered "No".

Regarding the body regions related to the subjective musculoskeletal symptoms of dental hygienists who answered "Yes", $79.9 \%$ had subjective musculoskeletal symptoms on the shoulders, $78 \%$ on the neck, and $66 \%$ on the wrists.

Regarding he question "do you turn your waist, or bend over in order for treatment?", 56.3\% answered "Yes", 29.5\% answered
"Yes, but not much", and 14.2\% answered "No".

Regarding the body regions related to the subjective musculoskeletal symptoms of dental hygienists who turned the waist or bent over for treatment, $79.6 \%$ had subjective musculoskeletal symptoms on the shoulders, $78.9 \%$ on the neck, $74.8 \%$ on the waist, and $66 \%$ on the wrists.

Regarding the question "do you sit on the edge of a chair for treatment, in terms of the hip posture?", 37.9\% answered "Yes", $29.5 \%$ answered "Yes, but not much", and 32.6\% answered "No".

Regarding the body regions related to the subjective musculoskeletal symptoms of dental hygienists who answered "Yes", $80.8 \%$ had subjective musculoskeletal symptoms on the shoulders, $76.8 \%$ on the neck, $72.7 \%$ on the waist, and $62.6 \%$ on the wrists.

Regarding the question "do you have break time between patients?", 13\% answered "Yes", 29\% answered "Yes, but for a moment", and 57.5\% answered "No".

Regarding the body regions related to the subjective musculoskeletal symptoms of dental hygienists whose break time

Table 3. Characteristics of treatment posture

\begin{tabular}{|c|c|c|c|c|c|c|c|c|c|c|c|}
\hline \multicolumn{2}{|c|}{ Variable } & \multirow{2}{*}{$\frac{N}{39}$} & \multirow{2}{*}{$\begin{array}{c}\text { Eye } \\
22 \text { (56.4) }\end{array}$} & \multirow{2}{*}{$\begin{array}{c}\text { Neck } \\
26(66.7)\end{array}$} & \multirow{2}{*}{$\begin{array}{c}\text { Shoulder } \\
34(87.2)\end{array}$} & \multirow{2}{*}{$\begin{array}{r}\text { Arm/elbow } \\
12(30.8)\end{array}$} & \multirow{2}{*}{$\begin{array}{l}\text { Hand } \\
12(30.8)\end{array}$} & \multirow{2}{*}{$\begin{array}{c}\text { Wrist } \\
25(64.1)\end{array}$} & \multirow{2}{*}{$\begin{array}{c}\text { Finger } \\
17(43.6)\end{array}$} & \multirow{2}{*}{$\begin{array}{c}\text { Waist } \\
30 \text { (76.9) }\end{array}$} & \multirow{2}{*}{$\begin{array}{c}\text { Leg/foot } \\
17(43.6)\end{array}$} \\
\hline Treatment & $\leq 2$ & & & & & & & & & & \\
\hline & $2-4$ & 121 & $47(38.8)$ & $91(75.2)$ & $92(76.0)$ & 37 (30.6) & $42(34.7)$ & 70 (57.9) & $36(29.8)$ & $86(71.1)$ & $81(66.9)$ \\
\hline & $\geq 4$ & 101 & 40 (39.6) & $74(73.3)$ & $78(77.2)$ & $31(30.7)$ & $42(41.6)$ & 68 (67.3) & $33(32.7)$ & $65(64.4)$ & $60(59.4)$ \\
\hline & $p$-value & & 0.131 & 0.578 & 0.328 & 1.000 & 0.400 & 0.339 & 0.278 & 0.300 & 0.033 \\
\hline \multirow{4}{*}{$\begin{array}{l}\text { The hand(s) } \\
\text { used }\end{array}$} & Right & 136 & $59(43.4)$ & $103(75.7)$ & $108(79.4)$ & $35(25.7)$ & $45(33.1)$ & $86(63.2)$ & $40(29.4)$ & 99 (72.8) & $88(64.7)$ \\
\hline & Left & 9 & 3 (33.3) & $6(66.7)$ & 7 (77.8) & 5 (55.6) & $5(55.6)$ & 7 (77.8) & $5(55.6)$ & 7 (77.8) & 7 (77.8) \\
\hline & Both & 116 & $47(40.5)$ & $82(70.7)$ & $89(76.7)$ & $40(34.5)$ & $46(39.7)$ & $70(60.3)$ & 41 (35.3) & $75(64.7)$ & $63(54.3)$ \\
\hline & p-value & & 0.785 & 0.602 & 0.876 & 0.083 & 0.276 & 0.561 & 0.207 & 0.323 & 0.136 \\
\hline \multirow{5}{*}{$\begin{array}{c}\text { Operation } \\
\text { position }\end{array}$} & 6 o'clock & 15 & 7 (46.7) & $10(66.7)$ & $10(66.7)$ & $4(26.7)$ & $5(33.3)$ & $10(66.7)$ & $3(20.0)$ & $11(73.3)$ & $7(46.7)$ \\
\hline & 9 o'clock & 166 & 71 (42.8) & $126(75.9)$ & $133(80.1)$ & $54(32.5)$ & $65(35$ & 107 & $56(33.7)$ & $117(70.5)$ & $110(66.3)$ \\
\hline & 12 o'clock & 68 & $24(35.3)$ & 46 (67.6) & $51(75.0)$ & $18(26.5)$ & $21(30.9)$ & 38 (55.9) & $23(33.8)$ & $45(66.2)$ & $34(50.0)$ \\
\hline & etc. & 12 & 7 (58.3) & $9(75.0)$ & $10(83.3)$ & 4 (33.3) & $5(41.7)$ & $8(66.7)$ & 4 (33.3) & $8(66.7)$ & 7 (58.3) \\
\hline & $p$-value & & 0.433 & 0.566 & 0.548 & 0.804 & 0.655 & 0.628 & 0.751 & 0.902 & 0.083 \\
\hline \multirow[t]{4}{*}{ Body in order } & Agree & 159 & 69 (43.4) & $124(78.0)$ & 127 (79.9) & 55 (34.6) & 65 (40.9) & $105(66.0)$ & $59(37.1)$ & $116(73.0)$ & 101 (63.5) \\
\hline & Neutral & 83 & 33 (39.8) & $56(67.5)$ & 63 (75.9) & $24(28.9)$ & $27(32.5)$ & $50(60.2)$ & $21(25.3)$ & $55(66.3)$ & $48(57.8)$ \\
\hline & Disagree & 19 & 7 (36.8) & $11(57.9)$ & $14(73.7)$ & $1(5.3)$ & $4(21.1)$ & $8(42.1)$ & 6 (31.6) & $10(52.6)$ & $9(47.4)$ \\
\hline & p-value & & 0.779 & 0.064 & 0.689 & 0.030 & 0.148 & 0.111 & 0.177 & 0.146 & 0.328 \\
\hline \multirow[t]{4}{*}{ Waist posture } & Agree & 147 & 60 (40.8) & $116(78.9)$ & 117 (79.6) & $48(32.7)$ & $57(38.8)$ & $97(66.0)$ & $50(34.0)$ & $110(74.8)$ & 91 (61.9) \\
\hline & Neutral & 77 & 32 (41.6) & $50(64.9)$ & 59 (76.6) & $25(32.5)$ & $28(36.4)$ & $44(57.1)$ & $24(31.2)$ & 49 (63.6) & $48(62.3)$ \\
\hline & Disagree & 37 & 17 (45.9) & $25(67.6)$ & $28(75.7)$ & 7 (18.9) & $11(29.7)$ & $22(59.5)$ & $12(32.4)$ & $22(59.5)$ & 19 (51.4) \\
\hline & p-value & & 0.851 & 0.057 & 0.812 & 0.248 & 0.592 & 0.397 & 0.909 & 0.084 & 0.466 \\
\hline \multirow[t]{4}{*}{ Hip posture } & Agree & 99 & $42(42.4)$ & $76(76.8)$ & $80(80.8)$ & $28(28.3)$ & 35 (35.4) & $62(62.6)$ & $30(30.3)$ & $72(72.7)$ & $56(56.6)$ \\
\hline & Neutral & 77 & 32 (41.6) & $51(66.2)$ & $58(75.3)$ & $28(36.4)$ & 32 (41.6) & 43 (55.8) & $24(31.2)$ & $51(66.2)$ & $48(62.3)$ \\
\hline & Disagree & 85 & 35 (41.2) & $64(75.3)$ & 66 (77.6) & $24(28.2)$ & $29(34.1)$ & $58(68.2)$ & 32 (37.6) & $58(68.2)$ & $54(63.5)$ \\
\hline & p-value & & 0.985 & 0.255 & 0.676 & 0.432 & 0.576 & 0.266 & 0.529 & 0.627 & 0.584 \\
\hline \multirow[t]{4}{*}{ Break time } & Agree & 34 & $13(38.2)$ & 23 (67.6) & $24(70.6)$ & $10(29.4)$ & $12(35.3)$ & 19 (55.9) & $15(44.1)$ & $20(58.8)$ & 18 (52.9) \\
\hline & Neutral & 77 & $31(40.3)$ & 55 (71.4) & $62(80.5)$ & $23(29.9)$ & $29(37.7)$ & $51(66.2)$ & $25(32.5)$ & 53 (68.8) & $42(54.5)$ \\
\hline & Disagree & 150 & $65(43.3)$ & $113(75.3)$ & $118(78.7)$ & 47 (31.3) & $55(36.7)$ & $93(62.0)$ & $46(30.7)$ & $108(72.0)$ & $98(65.3)$ \\
\hline & p-value & & 0.820 & 0.605 & 0.493 & 0.961 & 0.971 & 0.575 & 0.320 & 0.320 & 0.181 \\
\hline
\end{tabular}

Values are presented as number (\%). 
was short, $78.7 \%$ had subjective musculoskeletal symptoms on the shoulders, $75.3 \%$ on the neck, $72 \%$ on the waist, and $65.3 \%$ on the legs/feet [1]. Table 3 shows the distribution made according to the study subject's characteristics of treatment posture.

\section{Discussion}

This study analyzed the general treatment characteristics of dental hygienists working in dental clinical settings, the characteristics of their treatment conditions, and their posture for treatment. The analyzed results are as follows:

Regarding the characteristics of the dental institutions, they work for, most of them (83.1\%) worked at dental clinics. Regarding the body regions related to the subjective musculoskeletal symptoms of the dental hygienists working at dental clinics, $77.9 \%$ had subjective musculoskeletal symptoms on the shoulders, $75.1 \%$ on the neck, and $68.2 \%$ on the waist. As for those working at dental hospitals, $80.6 \%$ had subjective musculoskeletal symptoms on the shoulders, $72.2 \%$ on the waist, and $63.9 \%$ on the neck.

Regarding service career, dental hygienists with a career of 5 years accounted for $72.4 \%$. Among those with a career of less than 2 years, $77.7 \%$ had subjective musculoskeletal symptoms on the shoulders, $75.5 \%$ on the neck, and $74.5 \%$ on the waist. Among those with a career of 3 to 5 years, $78.9 \%$ had subjective musculoskeletal symptoms on the shoulders, $70.5 \%$ on the waist, and $68.4 \%$ on the neck. They had more subjective musculoskeletal pains on the waist, than on the neck.

Regarding of a type of work, $90.0 \%$ worked in treatment room. Among them, 79.1\% had subjective musculoskeletal symptoms on the shoulders, $73.6 \%$ on the neck, and $71.5 \%$ on the waist.

Their treatment conditions were analyzed. Regarding their medical subjects in which they are directly involved for treatment, $87.3 \%$ were involved in scaling, $78.5 \%$ in dental prosthetic treatment, and $65.9 \%$ in periodontal treatment. Sealant $(22.2 \%)$ as preventive treatment was relatively low. Regarding the treatment subjects directly involved in, scaling (87.3\%) accounted for the highest rate. Regarding the body regions related to their subjective musculoskeletal symptoms, $92.6 \% \mathrm{had}$ subjective musculoskeletal symptoms on the wrists, $90.0 \%$ on the waist, $89.4 \%$ on the fingers, $88.6 \%$ on the arms/elbows, $88.4 \%$ on the hands, $88.4 \%$ on the neck, $88.2 \%$ on the shoulders, $88.1 \%$ in the eyes, and $87.3 \%$ on the legs/feet. Subjective musculoskeletal symptoms were found in all body regions.

Regarding the count of weekly scaling, $49.0 \%$ performed scaling 1 to 5 times, $32.1 \%$ performed scaling 6 to 10 times, and $18.8 \%$ performed scaling more than 11 times.

In case of the groups of dental hygienists who performed scaling 1 to 5 times, and 6 to 10 times, their subjective muscu- loskeletal symptoms were most found in the order of the shoulders, the neck, and the waist. In case of the group of those who performed scaling more than 11 times, subjective musculoskeletal symptoms were found in the order of the neck, the shoulders, and the waist. In short, subjective musculoskeletal symptoms on the neck were more found.

Milerad et al. [12] reported that dental hygienists' chronic musculoskeletal disorders were related to their repeated dental work.

Regarding their daily treatment hours, those working 2 to 4 hours accounted for the highest rate $(46.4 \%)$, and their subjective musculoskeletal symptoms were found in the order of the shoulders $(76.0 \%)$, the neck $(75.2 \%)$, and the waist $(71.1 \%)$.

Regarding the use of their hands for treatment, those using both hands accounted for $44.4 \%$, and their subjective musculoskeletal symptoms were found in the order of the shoulders (76.7\%), the neck (70.7\%), and the waist (64.7\%), and the wrists $(60.3 \%)$. Subjective musculoskeletal symptoms on the wrists were more found.

Regarding dental hygienists' operation position for treatment, those in the 9 o'clock position accounted for $63.6 \%$, and their subjective musculoskeletal symptoms were found in the order of the shoulders $(80.1 \%)$, the neck $(75.9 \%)$, and the legs/feet $(66.3 \%)$.

Regarding the posture for treatment, $60.9 \%$ answered that they bent over more than 15 degrees or twisted their body. As a result, it was found that dental hygienists had the problem with posture for treatment [13].

Milerad et al. [12] reported that turning one's head in one side or lowering one's head over 20 degrees during oral treatment was the main cause of the muscle stress around the neck and waist. Regarding their waist posture for treatment, 56.3\% answered that they bent over or turned their waist. As a result, it was found that dental hygienists had the problem with waist posture for treatment.

Regarding their hip posture for treatment, $37.9 \%$ answered that they sit on the edge of a chair. Dental hygienists had relatively correct hip posture more than waist posture but had the problem with other kinds of posture.

Most dental hygienist had the problem with waist and shoulder posture for direct treatment, and suffered subjective musculoskeletal symptoms mostly on the shoulders, the neck, and the waist in order.

\section{Conclusion}

\section{General characteristics}

1) Regarding the characteristics of the dental institutions, they 
work for, most of them (83.1\%) worked at dental clinics-77.9\% had subjective musculoskeletal symptoms on the shoulders, $75.1 \%$ on the neck, and $68.2 \%$ on the waist. As for those working at dental hospitals, $80.6 \%$ had subjective musculoskeletal symptoms on the shoulders, $72.2 \%$ on the waist, and $63.9 \%$ on the neck.

2) Regarding of a type of work, $90 \%$ worked in treatment room-79.1\% had subjective musculoskeletal symptoms on the shoulders, $73.6 \%$ on the neck, and $71.5 \%$ on the waist.

3) Regarding service career dental hygienists with a career of less than 2 years, $77.7 \%$ had subjective musculoskeletal symptoms on the shoulders, $75.5 \%$ on the neck, and $74.5 \%$ on the waist. Among those with a career of 3 to 5 years, $78.9 \%$ had subjective musculoskeletal symptoms on the shoulders, $70.5 \%$ on the waist, and $68.4 \%$ on the neck. They had more subjective musculoskeletal pains on the waist, than on the neck.

\section{Characteristics of treatment}

Regarding the count of weekly scaling, $49.0 \%$ performed scaling 1 to 5 times, $32.1 \%$ performed scaling 6 to 10 times, and $18.8 \%$ performed scaling more than 11 times.

In case of the group of those who performed scaling more than 11 times, subjective musculoskeletal symptoms were found in the order of the neck, the shoulders, and the waist. In short, subjective musculoskeletal symptoms on the neck were more found.

\section{Characteristics of treatment posture}

1) Regarding their daily treatment hours, those working 2 to 4 hours accounted for the highest rate (46.4\%)-Their subjective musculoskeletal symptoms were found in the order of the shoulders $(76.0 \%)$, the neck $(75.2 \%)$, and the waist $(71.1 \%)$.

2) Regarding the use of their hands for treatment, those using both hands accounted for $44.4 \%$-Their subjective musculoskeletal symptoms were found in the order of the shoulders (76.7\%), the neck $(70.7 \%)$, and the waist $(64.7 \%)$, and the wrists $(60.3 \%)$. Subjective musculoskeletal symptoms on the wrists were more found.

3) Regarding dental hygienists' operation position for treatment, those in the 9 o' clock position accounted for $63.6 \%$-Their subjective musculoskeletal symptoms were found in the order of the shoulders $(80.1 \%)$, the neck $(75.9 \%)$, and the legs/feet $(66.3 \%)$.

4) Regarding the posture for treatment, $60.9 \%$ answered that they bent over more than 15 degrees or twisted their body.

5) Regarding their waist posture for treatment, 56.3\% answered that they bent over or turned their waist.

6) Regarding their hip posture for treatment, $37.9 \%$ answered that they sit on the edge of a chair.
Dental hygienists had relatively correct hip posture more than waist posture but had the problem with other kinds of posture.

\section{Conflict of Interest}

No potential conflict of interest relevant to this article was reported.

\section{ORCID}

hyo jeong kim, https://orcid.org/0000-0002-5407-2718

\section{References}

1. Jeon SH, Han GS. A study on dental hygienist environmental working conditions and subjective pain symptoms. J Korean Acad Oral Health 2011;35:347-59.

2. National Institute for Occupational Safety and Health. Musculoskeletal disorders and workplace factors: a critical review of epidemiologic evidence for work-related musculoskeletal disorders of the neck, upper extremity, and low back. Cincinnati: NIOSH; 1997.

3. Nield-Gehrig JS. Fundamentals of periodontal instrumentation \& advanced root instrumentation. 6th ed. Jong WK, translator. Seoul: Daehannarae Publishing; 2009:11-7.

4. Sanders MJ, Michalak-Turcotte C. Preventing work-related MSDs in dental hygienists. In: Sanders MJ, ed. Ergonomics and the management of musculoskeletal disorders. 2nd ed. St. Louis: Butterworth Heinemann; 2004:448-73.

5. Morse T, Bruneau H, Michalak-Turcotte C, Sanders M, Warren N, Dussetschleger J, et al. Musculoskeletal disorders of the neck and shoulder in dental hygienists and dental hygiene students. J Dent Hyg 2007;81:10.

6. Oh EJ, Hwang SJ. Comparison of the working conditions of dental hygienists using data from online job sites. J Dent Hyg Sci 2017;17:501-7.

7. Jeong KY, Han OS. A study on emotional labor, emotional burnout, turnover intention of dental hygienist. J Dent Hyg Sci 2015; 15: 280-6.

8. Park JH, Lee YH. Influence of dental hygienists' core competencies on job performance. J Dent Hyg Sci 2017;17:142-9.

9. Åkesson I, Balogh I, Hansson GÅ. Physical workload in neck, shoulders and wrists/hands in dental hygienists during a work-day. Appl Ergon 2012;43:803-11.

10. Silverstein BA, Fine LJ, Armstrong TJ. Hand wrist cumulative trauma disorders in industry. Br J Ind Med 1986;43:779-84.

11. Alexopoulos EC, Stathi IC, Charizani F. Prevalence of musculoskeletal disorders in dentists. BMC Musculoskelet Disord 2004;5:16.

12. Milerad E, Ericson MO, Nisell R, Kilbom A. An electromyographic study of dental work. Ergonomics 1991;34:953-62.

13. Lalumandier JA, McPhee SD, Parrott CB, Vendemia M. Musculoskeletal pain: prevalence, prevention, and differences among dental office personnel. Gen Dent 2001;49:160-6. 